\title{
APENDICECTOMÍA LAPAROSCÓPICA VERSUS CLÁSICA EN PACIENTES OBESOS*
}

\author{
Dr. Marcelo Zamorano D. ${ }^{1}$
}

1 Servicio de Cirugía. Hospital Regional de Antofagasta Dr. Leonardo Guzmán. Facultad de Medicina, Universidad de Antofagasta.

Antofagasta, Chile.

\author{
TRABAJO DE INGRESO
}

\section{Abstract}

\section{Comparison of open and laparoscopic surgery in obese patients}

Background: Laparoscopic appendectomy may have advantages over the open surgical technique in obese patients. Aim: To compare the results and complications of open and laparoscopic appendectomy in obese patients. Material and Methods: Patients consulting for acute appendicitis with a body mass index of $30 \mathrm{~kg} / \mathrm{m}^{2}$ or more were randomly assigned to be treated by open of laparoscopic appendectomy. Both surgical techniques were standardized. Results: Seventy patients were studied. Half were treated with laparoscopic and half with open surgery. Demographic variables were comparable in both groups. Surgical times for open and laparoscopic appendectomies were 35 and $42 \mathrm{~min}$ respectively. The figures for hospital stay were 2.5 and 2.1 days respectively. Surgical wound problems more common in open surgery. Conversion to open surgery occurred in 3\% of patients. Conclusions: In this group of obese patients, laparoscopic appendectomy had a low rate of conversion and lesser wound complications

Key words: Appendectomy, open surgery, laparoscopic surgery.

\section{Resumen}

Introducción: Desde la introducción de la cirugía laparoscópica la apendicectomía se ha considerado un punto controversial. Existen estudios que establecen ventajas de la apendicectomía laparoscópica en pacientes obesos. Nuestro objetivo fue comparar los resultados y complicaciones postoperatorias en pacientes con Índice de Masa Corporal (IMC) mayor o igual a 30, atendidos en el Hospital Regional de Antofagasta. Material y Métodos: Realizamos un estudio prospectivo aleatorio, mediante método computacional de probabilidad uniforme. Se incluyó consecutivamente a todos los pacientes con diagnóstico de apendicitis

*Recibido el 20 de mayo de 2014 y aceptado para publicación el 29 de junio de 2015.

Autor declara no tener conflictos de interés.

La casuística fue desarrollada en Antofagasta. En la actualidad, el autor está realizando una Beca de Cirugía Digestiva en el Departamento de Cirugía del Hospital Clínico de la Universidad de Chile.

Correspondencia: Dr. Marcelo Zamorano D. zamoranomarcelo@gmail.com 
aguda e IMC mayor o igual a 30, desde el $1^{\circ}$ de julio de 2010 al 30 de abril de 2012. Se protocolizaron ambas técnicas quirúrgicas. Se utilizó estadística descriptiva y $\chi^{2}$. Resultados: Se incluyeron 70 pacientes, no hubo diferencias estadísticamente significativas en las variables demográficas de ambos grupos. En cuanto al tiempo quirúrgico en técnica abierta y laparoscópica, obtuvimos una media de 35 y 42 min respectivamente, sin diferencias estadísticamente significativas. Los días de estada fueron de 2,5 y 2,1 respectivamente con un $p>0,05$. En cuanto a las complicaciones locales incluyendo seroma e infección de herida operatoria, fueron mayores en el grupo de apendicectomía abierta, estadísticamente significativo $(\mathrm{p}<0,05)$. Se convirtió de laparoscopia a técnica abierta en un 2,9\% de los casos. Conclusiones: En este estudio, la apendicectomía laparoscópica obtiene buenos resultados, con bajo índice de conversión y con menos complicaciones locales en pacientes obesos.

Palabras clave: Apendicectomía laparoscópica, apendicectomía abierta, complicaciones, obesidad.

\section{Introducción}

La apendicitis aguda constituye la entidad quirúrgica más frecuentemente intervenida por el cirujano general en los servicios de emergencia. En nuestro país la apendicectomía es la cirugía digestiva por patología benigna más frecuente, con 30.997 cirugías en el período 2005 a 2006, seguida por la colecistectomía con 29.850 operaciones y por la herniorrafia con 23.519 operaciones durante el mismo período, datos referentes a los Hospitales del Ministerio de Salud ${ }^{1,2}$.

La técnica estándar para el tratamiento de la apendicitis, hasta hace algunos años, fue la descrita por McBurney en 1894³. En 1982, Semm publicó los primeros casos de apendicitis tratados por vía laparoscópica y desde entonces ha estado en discusión cuál es la técnica más beneficiosa para el paciente ${ }^{4}$. Debido a la reducción del tiempo de estancia postoperatoria, menor dolor y menos complicaciones en el sitio operatorio, la técnica por vía laparoscópica ha ganado cada vez más adeptos ${ }^{3,4}$. Sin embargo, la literatura hace mención a factores adversos asociados a esta técnica, como abscesos intraabdominales y mayor costo operatorio 5 .

El problema de la obesidad y el sobrepeso afectan a una gran parte de la población adulta en nuestro país, constituyendo un problema de salud pública relevante ${ }^{6}$. Por lo tanto, es necesario considerar los tratamientos de estos pacientes para poder determinar mejoras en los servicios de atención pública. El grosor de la pared abdominal de los pacientes obesos constituye una dificultad en términos de acceso y la laparoscopia puede superar este obstáculo con facilidad, lo que lleva a postular una eventual superioridad de esta técnica? ${ }^{7}$.

El objetivo del presente estudio fue analizar los resultados clínicos en pacientes sometidos a apendicectomía abierta (AA) y laparoscópica (AL) en población obesa, clasificados por el IMC, para observar si existen o no ventajas de una técnica sobre otra, exponiendo nuestra realidad nacional.

\section{Materiales y Método}

\section{Pacientes estudiados}

Se realizó un estudio prospectivo, aleatorio entre apendicectomía laparoscópica y abierta en pacientes obesos en el Hospital Regional de Antofagasta Dr. Leonardo Guzmán (HRA), durante el período comprendido entre el $1^{\circ}$ de julio de 2010 y el 30 de abril de 2012.

Se incluyó pacientes que consultaron en el Servicio de Emergencias de Adultos del HRA, que comprenden la población con edad mayor o igual de 15 años. Se enrolaron sólo los pacientes con IMC $\geq$ 30 con diagnóstico de apendicitis aguda, con o sin peritonitis apendicular, obteniéndose una muestra de 70 enfermos, 35 por rama. Se excluyó a pacientes con diagnóstico de abdomen agudo explicado por otra causa.

De todos los pacientes se obtuvo consentimiento informado, tanto el perteneciente al HRA como el del estudio a realizar.

\section{Método}

Los pacientes con dolor abdominal que ingresaron al servicio de urgencias fueron evaluados por los cirujanos autores de este trabajo, quienes realizaron los procedimientos quirúrgicos. Cabe destacar que la indicación quirúrgica de los pacientes se realizó según la pauta clásica de diagnóstico clínico9, requiriendo exámenes de laboratorio e imagen en algunos casos.

Se formaron 2 grupos, el grupo control formado por 35 pacientes que fueron sometidos a AA y el grupo estudio, que fue formado por igual número de pacientes sometidos a AL. Se aleatorizó mediante método computacional de distribución de probabilidad uniforme.

\section{Técnica quirúrgica}

Las técnicas quirúrgicas se estandarizaron y se realizaron por los mismos cirujanos (2) en todos los casos. 
La AL se realizó con 3 trocares, dos de $10 \mathrm{~mm}$, uno en ombligo, otro en fosa ilíaca derecha por fuera de la vaina de los rectos y uno de $5 \mathrm{~mm}$ en hipogastrio a izquierda por fuera de los vasos epigástricos inferiores. El procedimiento se realizó en forma similar a la AA, esta última se realizó con incisión de McBurney. No se utilizó jareta.

Respecto a la operación, se determinaron tiempo operatorio, diagnóstico intraoperatorio y conversión. Posterior a la cirugía se evaluó días de hospitalización y complicaciones, este último parámetro se valoró mediante tres controles ambulatorios. El primero se realizó a los 7 días, el segundo al mes, y el tercero al segundo mes postoperatorio.

\section{Cálculo estadístico}

Se realizaron análisis mediante el software XLSTAT $9 \circledR$. Las complicaciones que se consideraron para el estudio fueron: seroma, infección superficial de herida operatoria y dehiscencia de la sutura (piel). Se analizaron y compararon las variables mediante estadística descriptiva y prueba de $\chi^{2}$.

\section{Definiciones}

1. Apendicitis aguda: proceso inflamatorio agudo del apéndice cecal producido por obstrucción del lumen y proliferación bacteriana subsiguiente.

a. Apendicitis edematosa: el apéndice muestra hiperemia y edema sin o con escaso exudado seroso.

b Apendicitis flegmonosa: el apéndice y el mesoapéndice están edematosos, presenta petequias y láminas de exudado fibrinopurulento. No hay compromiso de la muscular.

c. Apendicitis necrótica: se agrega áreas moradas, verdes grisáceas o negras. Presenta microperforaciones, líquido peritoneal purulento, la capa muscular se encuentra necrótica.

d. Apendicitis perforada: Hay ruptura macroscópica.

2. Seroma: colección de líquido seroso dentro de la cavidad residual postquirúrgica.
3. Infección de herida operatoria: se consideró la infección incisional superficial que cumple las siguientes condiciones:

a. Ocurre los primeros 30 días postoperatorios, se manifiesta por dolor, inflamación, eritema y calor.

b. Compromete únicamente la piel y los tejidos blandos subcutáneos a la incisión.

c. Puede presentar: drenaje purulento, con o sin confirmación microbiológica.

d. No compromete músculo ni fascia.

4. Reoperación: realización de una operación no planificada a un paciente ya operado como consecuencia de la cirugía primaria dentro de los 30 días postoperatorios.

5. Complicaciones:

a. Precoces: Se presentan antes del alta.

b. Tardíos: Se presentan posterior al alta.

\section{Resultados}

Se incluyeron 70 casos. De los pacientes sometidos a AA $13(37 \%)$ eran hombres y 22 (63\%) mujeres, mientras que los pacientes tratados con $\mathrm{AL}$ $11(31,4 \%)$ fueron hombres y $24(68,6 \%)$ mujeres. La edad media del grupo de AA fue de 32 años con desviación estándar poblacional (DEP) de $32 \pm 11,3$ años. El grupo de AL mostró una media de 30,02 años con una DEP de $30 \pm 10$. El IMC promedio dentro del grupo tratado con AA fue 33,6 y DEP $33,6 \pm 2,5$, paralelamente el grupo abordado vía laparoscópica tuvo un IMC promedio de 34,6 con DEP de 34,6 $\pm 3,8$ (Tabla 1).

No hubo diferencias significativas en las variables anteriormente descritas lo que hace a estos dos grupos comparables $(\mathrm{p}>0,05)$.

En cuanto a la estancia postoperatoria no hubo diferencias significativas $(p>0,05)$. La media aritmética en el grupo con AA fue 2,45 días (rango 1-4 días), mientras que en el grupo con AL fue 2,17

Tabla 1. Distribución de la población por sexo, edad e IMC

\begin{tabular}{|lcccc|}
\hline & Laparoscopia $\mathbf{n}=\mathbf{3 5}$ & Clásica $\mathbf{n}=\mathbf{3 5}$ & $\mathbf{p}$ \\
Sexo & Masculino & 11 & 13 & NS \\
& Femenino & 24 & 22 & NS \\
Edad: Media (DE) & $30( \pm 10,0)$ & $32( \pm 11,3)$ & NS \\
IMC $\left(\mathrm{kg} / \mathrm{m}^{2}\right)$ Media $(\mathrm{DE})$ & $34,6( \pm 3,8)$ & $33,6( \pm 2,5)$ & NS \\
\hline
\end{tabular}

NS: No significativo. Significativo con $\mathrm{p}<0,05$. IMC: Índice de masa corporal. Media aritmética. DE: Desviación estándar poblacional. 
Tabla 2. Tiempos quirúrgicos y estancia hospitalaria

\begin{tabular}{|lccc|}
\hline & Laparoscopia $\mathbf{n}=\mathbf{3 5}$ & Clásica $\mathbf{n}=\mathbf{3 5}$ & $\mathbf{p}$ \\
\hline Tiempo quirúrgico: minutos (rango) & $41,8(25-60)$ & $35,18(20-50)$ & NS \\
\hline Días de hospitalización: días (rango) & $2,17(1-4)$ & $2,45(1-4)$ & NS \\
\hline
\end{tabular}

NS: No significativo. Significativo con $p<0,05$.

Tabla 3. Tipo de apendicitis aguda

\begin{tabular}{|cccc|}
\hline Diagnóstico & Laparoscopia n $=\mathbf{3 5}$ & Clásica $\mathbf{n}=\mathbf{3 5}$ & $\mathbf{p}$ \\
\hline 1 Edematosa & $7(20 \%)$ & $5(14,3 \%)$ & NS \\
\hline 2 Flegmonosa & $12(34,3 \%)$ & $16(45,7 \%)$ & NS \\
\hline 3 Necrótica & $11(31,4 \%)$ & $8(22,8 \%)$ & NS \\
\hline 4 Perforada & $5(14,3 \%)$ & $6(17,4 \%)$ & NS \\
\hline
\end{tabular}

NS: No significativo.

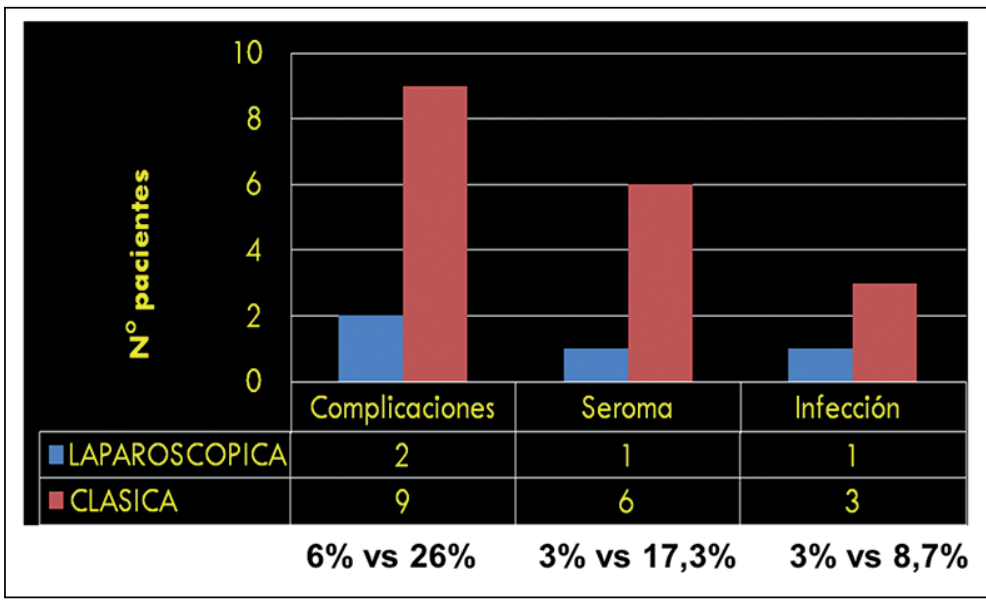

Figura 1. Incidencia de complicaciones después de apendicectomía abierta y laparoscópica en pacientes con IMC igual o mayor de $30 \mathrm{~kg} / \mathrm{m}^{2}$. días con igual rango que el grupo anterior. El tiempo operatorio fue mayor en el abordaje laparoscópico con 41,8 min (rango 25-60 min) vs 35,18 min (rango 20-50 min) en la técnica abierta, sin embargo, no existen diferencias estadísticamente significativas $(p>0,05)$ (Tabla 2). El diagnóstico intraoperatorio de las dos técnicas se resume en la Tabla 3. Fueron más frecuentes los apéndices flegmonosos en ambos grupos con $16(45,7 \%)$ y $12(34,3 \%)$, tanto en el grupo con abordaje abierto y laparoscópico respectivamente. En cuanto a los pacientes con peritonitis apendicular se constató el diagnóstico en 6 y 5 pacientes, en AL y AA respectivamente. No se evidenció pacientes con absceso apendicular en ningún grupo.
No hubo mortalidad asociada. En los pacientes sometidos a la AA se presentaron 9 complicaciones (26\%): 6 seromas y 3 infecciones de la herida operatoria. Por otro lado, los pacientes intervenidos por vía laparoscópica presentaron 2 (6\%) complicaciones: 1 seroma y 1 infección de herida (Figura 1). Se realizó sólo una conversión al realizar la AL. La incidencia de complicaciones locales en pacientes tratados por técnica abierta evidenció diferencias estadísticamente significativas respecto al grupo tratado con AL $(\mathrm{p}<0,05)$ (Tabla 4).

Respecto a la asistencia de los pacientes a los controles propuestos, se observó una asistencia del $100 \%$ al primer control en ambos grupos y posteriormente una asistencia similar y adecuada. 
Tabla 4. Complicaciones postoperatorias y conversiones

\begin{tabular}{|cccc|}
\hline & Laparoscopia $\mathbf{n}=\mathbf{3 5}$ & Clásica $\mathbf{n}=\mathbf{3 5}$ & $\mathbf{p}$ \\
Complicaciones totales & $2(6 \%)$ & $9(26 \%)$ & $\mathrm{p}<0,05^{*}$ \\
Seroma & $1(3 \%)$ & $6(17,3 \%)$ & \\
Infección & $1(3 \%)$ & $3(8,7 \%)$ & \\
Reoperación & 0 & 0 & \\
Conversiones & $1(2,9 \%)$ & & \\
\hline
\end{tabular}

*Estadísticamente Significativo.

Tabla 5. Complicaciones en apendicectomía laparoscópica, resultados nacionales

\begin{tabular}{|c|c|c|c|c|c|c|}
\hline Autor & Lugar de estudio y año & $\begin{array}{c}\text { n pacientes } \\
\text { AL }\end{array}$ & $\begin{array}{c}\text { n complicaciones } \\
\text { precoces }\end{array}$ & $\%$ & $\begin{array}{c}\text { n complicaciones } \\
\text { tardías }\end{array}$ & $\%$ \\
\hline Rivera y cols. & $\begin{array}{l}\text { Hospital Naval } \\
\text { Punta Arenas } 1998\end{array}$ & 40 & 0 & 0 & 0 & 0 \\
\hline Rivera y cols. & $\begin{array}{l}\text { Hospital Naval } \\
\text { Punta Arenas } 2001\end{array}$ & 30 & 1 & 3,3 & 3 & 10 \\
\hline Vallejos y cols & $\begin{array}{l}\text { Hospital Regional } \\
\text { de Coyhaique } 2006\end{array}$ & 40 & 1 & 2,5 & 7 & 17,5 \\
\hline Zamorano y cols. & $\begin{array}{l}\text { Hospital Regional } \\
\text { de Antofagasta } 2012\end{array}$ & 35 & 2 & 5,7 & 0 & 0 \\
\hline
\end{tabular}

$\mathrm{N}^{\mathrm{o}}=$ número de complicaciones en la serie. $\%=$ porcentaje de complicaciones en la serie. $\mathrm{AL}=$ Apendicectomía laparoscópica.

\section{Discusión}

La apendicitis aguda es una de las causas más frecuentes de consulta quirúrgica en los servicios de urgencia nacionales ${ }^{8,9}$. De igual manera, la obesidad es una enfermedad con una prevalencia cada vez mayor tanto en Chile como a nivel mundial, es por eso que la obesidad ha sido ampliamente estudiada en la literatura como factor de riesgo que afectaría los resultados operatorios y postoperatorios ${ }^{10,11}$. Sumado a lo anterior, los cambios epidemiológicos que ha presentado la apendicitis aguda con la disminución sostenida de la mortalidad y morbilidad a nivel mundial y nacional, como lo reportara ya en 1991 De la Fuente y cols., permite considerar otros aspectos a la hora de elegir un abordaje quirúrgico, como la mínima invasión o la incidencia de complicaciones $^{12}$.

Los beneficios de la cirugía laparoscópica en obesos parecen ser más concluyentes que en la población general. El mayor espesor de la pared abdominal podría estar asociado a tasas de infecciones más altas en el caso de una laparotomía, lo que conlleva tiempos de recuperación prolongados y mayor estancia hospitalaria ${ }^{6}$. Sin embargo, la variabilidad de resultados entre estudios, así como las metodologías y la calidad de los mismos impide generar una conclusión concreta.
La apendicectomía laparoscópica se ha extendido a muchos centros quirúrgicos, sin embargo, se ha asociado a tiempo quirúrgico más prolongado y a la necesidad de mayor equipamiento. Contradiciendo lo primero, el estudio retrospectivo de Corneille y cols. (2007), en pacientes obesos, muestra tiempos quirúrgicos favorables para los pacientes tratados con AL con una media de $80 \mathrm{~min}$ vs $118 \mathrm{~min}$ de la AA $(p<0,001)^{13}$. Nuestro estudio no mostró diferencias significativas entre ambos abordajes, lo que concuerda con la serie descrita por Clarke y cols. (2011), estudio prospectivo de igual objetivo, donde tampoco hubo diferencias estadísticamente significativas ${ }^{7}$. En nuestro estudio se evidenció una reducción del tiempo operatorio realizando AL desde el inicio hacia el final del período, lo que estaría dado por la curva de aprendizaje y el uso de la técnica protocolizada.

En cuanto a la estadía hospitalaria difiere entre estudios prospectivos y retrospectivos. La revisión de Mason y cols. (2012), de la base de datos de American College of Surgeons National Surgical, evidencia menores tiempos de hospitalización en pacientes sometidos a AL con una diferencia de 1,2 días respecto del grupo tratado con $\mathrm{AA}^{14}$. También el estudio retrospectivo de Markar y cols. (2011), sostiene resultados similares ${ }^{15}$. En contraste con estas diferencias, el presente estudio, como la serie 
de Clarke y cols. (2011), no encontró variaciones significativas en este parámetro ${ }^{7}$.

Al igual que en esta experiencia, la serie de Varela y cols. (2008), en pacientes con obesidad mórbida, se encontraron diferencias significativas en cuanto a las complicaciones, siendo más frecuente las infecciones en el sitio operatorio ${ }^{16}$. Este y otros estudios concluyen resultados similares, razón por la cual estamos más cerca de afirmar la superioridad de la $\mathrm{AL}$ en este sentido ${ }^{13,14,16}$.

Las diferencias entre las investigaciones de los parámetros mencionados anteriormente, pueden explicarse debido a la experiencia de los cirujanos operando a la población obesa. Esto afectaría mayormente al tiempo operatorio, tasas de infección en la herida operatoria y el dolor postoperatorio, mientras que la experiencia clínica tratando a estos pacientes podría afectar en la estancia hospitalaria ${ }^{17,18}$. En nuestro estudio se consideraron estos factores, por lo tanto, se estandarizó el procedimiento quirúrgico $\mathrm{y}$ todos los pacientes de nuestra serie fueron tratados por los autores.

El dolor postoperatorio es una variable considerada subjetiva, y el método de objetivarla difiere entre los diferentes estudios dificultando el análisis comparativo. La metodología más frecuente para evaluar el dolor postoperatorio es determinar la cantidad de analgésicos administrados en el postoperatorio. A pesar de los resultados a favor de la laparoscopia en este aspecto, Clarke y cols. (2011), no encontraron diferencias significativas en el uso de analgesia ${ }^{7,13,19}$.

\section{Experiencia nacional}

En nuestro medio la experiencia en AL ha sido reportada de forma creciente y con resultados alentadores, siendo destacable su progresiva implementación en servicios de urgencia de hospitales públicos $^{20-22}$.

Entre las experiencias nacionales (Tabla 5), en 1998 Rivera y cols, en el Hospital Naval de Punta Arenas, presentó resultados sobre 40 pacientes intervenidos, destacando tiempo operatorio de 75,25 min, (35-230 $\mathrm{min})$, estadía hospitalaria promedio de 2,28 días, (1-23 días), alta antes de $48 \mathrm{~h}$ en el $82,5 \%$ de los pacientes y ausencia de complicaciones $^{23}$.

Posteriormente, el mismo equipo reportó en 2001 su experiencia en peritonitis aguda apendicular entre 1995 y 1998, se intervinieron con AL a 30 pacientes, de los cuales se convirtió tres a cirugía abierta por dificultades técnicas, la estadía hospitalaria promedio fue de 2,9 días (1-9 días). No hubo infección de heridas operatorias en forma precoz ni mortalidad en la serie. El tiempo operatorio promedio fue de 76,6 min, (45-150 min). Se presentaron como complicaciones un hemoperitoneo que requirió reopera- ción, dos casos de fiebre durante el postoperatorio y un absceso intraabdominal ${ }^{24}$.

Ambos trabajos no registraron el IMC de los pacientes lo que nos impide hacer comparaciones, sin embargo, los tiempos de hospitalización, como el alta precoz fueron similares a nuestro estudio, además destaca la seguridad de la técnica en casos complicados como la peritonitis aguda. El tiempo operatorio fue mayor, probablemente por la curva de aprendizaje.

Respecto a las complicaciones asociadas a $\mathrm{AL}$, la incidencia nacional es comparable a los resultados internacionales, sin embargo, existe reporte de dos casos que presentaron absceso retroperitoneal como complicación ${ }^{25}$, cabe señalar que en ambos casos no destacó la obesidad dentro de las morbilidades asociadas, ambos pacientes presentaron apendicitis aguda no complicada y no presentaban alteraciones de la inmunidad, por lo que se planteó que esta complicación podía deberse al uso de neumoperitoneo en la técnica laparoscópica, sin embargo, en el resto de series nacionales no se ha reportado.

El año 2006 se publicó la experiencia en el Hospital Regional de Coyhaique reportada por Vallejos y cols., en el cual se intervinieron 176 pacientes, 40 AL, 136 AA. Ambos grupos fueron comparables, la edad media para AL fue de 31 años (15-82) y en AA 32 años (15-78). El tiempo quirúrgico de $\mathrm{AL}$ tuvo una media de $42,3 \mathrm{~min}$, mientras que en AA fue $46,3 \mathrm{~min}$ sin diferencia significativa ${ }^{8}$.

La infección de la herida operatoria se presentó en 1 paciente de AL y en 12 de AA, esto no fue significativo. Sin embargo, las complicaciones postoperatorias durante el alta fueron $7 / 40$ para AL vs $17 / 136$ para AA $(p<0,05)$. La ocurrencia de colecciones intraabdominales fue mayor en $\mathrm{AL}$ sin diferencia significativa. El tiempo en reiniciar alimentación vía oral fue significativamente menor en los pacientes sometidos a $\mathrm{AL}^{8}$.

Este estudio tuvo resultados similares a los nuestros en relación a rango etario, tiempo operatorio, incidencia de complicaciones y alta precoz, sin embargo, en su protocolo no se definió el IMC por lo que no podemos comparar resultados. Sin embargo, sus resultados concuerdan con la literatura mundial y establecen la seguridad de la AL y sus beneficios que la hacen comparable e incluso superior a la técnica abierta.

\section{Conclusiones}

Según los resultados de nuestra serie, y basados en la evidencia, la AL parece presentar ventajas sobre el abordaje clásico en términos de menores complicaciones locales, presentando similares 
tiempos operatorios y estancia hospitalaria. Por lo tanto, sugerimos el abordaje laparoscópico como tratamiento de la apendicitis en la población obesa, siempre y cuando la experiencia y el equipamiento quirúrgico lo permitan. No obstante, debido a la escasez de estudios metodológicamente adecuados, nos parece prudente comparar estos resultados con futuros estudios prospectivos, aleatorizados para determinar firmemente la superioridad o no de la $\mathrm{AL}$ frente a la AA.

\section{Referencias}

1. Godoy A, Itriago D, Machado T, Nieve A, Ramírez A. Apendicectomía abierta vs apendicectomía por laparoscopia. Experiencia en el servicio de cirugía uno. Rev Venez Cir. 2007;60:71-7.

2. Csendes A, González G. Cirugías digestivas más frecuentes en Chile. Rev. Chil Cir. 2008;60:379-86.

3. Shaikh AR, Sangrasi AK, Shaikh GA. Clinical outcomes of laparoscopic versus open appendectomy. JSLS 2009;13:574-80.

4. Kehagias I, Karamanakos S, Panagiotopoulos S, Panagopoulos K, Kalfarentzos F. Laparoscopic versus open appendectomy: Which way to go? World J Gastroenterol. 2008;14:4909-14.

5. Sauerland S, Lefering R, Neugebuer EA. Laparoscopic versus open surgery for suspected appendicitis. Cochrane Database Syst Rev. 2004;18:CD001546.

6. Ministerio de Salud. Encuesta nacional de salud ENS Chile 2009-2010.

7. Clarke T, Katkhouda N, Mason R, Cheng B, Olasky J, Sohn H, et al. Laparoscopic versus open appendectomy for the obese patient: a subset analysis from a prospective, randomized, double-blind study. Surg Endosc. 2011;25:1276-80.

8. Vallejos C. Apendicectomía laparoscópica vs apendicectomía abierta en apendicitis aguda. Experiencia del Hospital Regional de Coyhaique. Rev Chil Cir. 2006;58:114-21.

9. Coñoman H, Cavalla C, Reyes D, Morán J, Cúneo A, Jirón A. Apendicitis aguda en el Hospital San Juan de Dios. Rev Chil Cir. 1999;51:176-83.

10. Dindo D, Kuller MK, Weber M. Obesity in general elective surgery. Lancet 2003;361:2032-5.
11. Pasulka P, Bistrian P, Blackburn G. The risks of surgery in obese patients. Ann Intern Med. 1986;104:540-6.

12. De la Fuente H, Puga B, Braghetto I, Yentzen G. Características epidemiológicas de la apendicitis en Chile, Rev Chil Cir. 1991;43:412-8.

13. Corneille M, Steigelman M, Myers J, Jundt J, Dent D, López, et al. Laparoscopic appendectomy is superior to open appendectomy in obese patients. Am J Surg. 2007;194:877-81.

14. Mason R, Moazzez A, Moroney J, Katkhouda N. Laparoscopic vs Open Appendectomy in Obese Patients: Outcomes Using the American College of Surgeons National Surgical Quality Improvement Program Database. J Am Coll Surg. 2012;215:88-97.

15. Markar S, Venkat-Raman V, Ho A, Karthikesalingam A, Kinross J, Evans J, Bloom I. Laparoscopic versus open appendicectomy in obese patients. Int $\mathrm{J}$ Surg. 2011;9:451-5.

16. Varela JE, Hinojosa MW, Nguyen NT. Laparoscopy should be the approach of choice for acute appendicitis in the morbidly obese. Am J Surg. 2008;196:218-22.

17. Katkhouda N, Mason RJ, Towfigh S. Laparoscopic versus open appendectomy: a prospective randomized doubleblind study. Ann Surg. 2005;242:439-48.

18. Kapischke M, Caliebe A, Tepel J. Open versus laparoscopic appendicectomy: a critical review. Surg Endosc. 2006;20:1060-8.

19. Delaney CP, Pokala N, Senagore AJ. Is laparoscopic colectomy applicable to patients with body mass index $>30$ ? A case-matched comparative study with open colectomy. Dis Colon Rectum 2005;48:975-81.

20. Ovalle C, Farías C, Olivares P. Apendicectomía Laparoscópica. Rev Chil Cir. 1991;43:438-41.

21. Rivera C. Apendicectomía Laparoscópica en apendicitis aguda. Rev Chil Cir. 1998;50:208-15.

22. Larraechea P. Laparoscopia diagnóstica en sospecha de apendicitis aguda. Rev Chil Cir. 2000;52:227-34.

23. Rivera C. Apendicectomía laparoscópica en apendicitis aguda. Rev Chil Cir. 1998;50:208-15.

24. Rivera C, Ross M. Apendicectomía laparoscópica en peritonitis apendicular. Rev Chil Cir. 2001;53:186-9.

25. Sciaraffia C, Soto G. Mariani V, Gutiérrez L, Marín M. Absceso retroperitoneal: una complicación infrecuente de la apendicectomía laparoscópica. Rev Chil Cir. 2002;50:401-3. 


\section{INFORME TRABAJO DE INGRESO}

La apendicectomía es un procedimiento que se incorporó desde los inicios de la cirugía laparoscópica en Chile, hay reportes desde 1990 de su ejecución.

La apendicectomía clásica con la incisión de Mc Burney ha sido la operación más ampliamente y exitosamente utilizada.

La cirugía laparoscópica ha demostrado ser segura y beneficiosa en procedimientos como colecistectomía, cirugía antireflujo, colectomía, etc., por disminución de tiempo de hospitalización, dolor postoperatorio, complicaciones y costos.

Existen numerosos reportes comparativos entre la apendicectomía clásica y laparoscópica, no logrando demostrar un beneficio claro y completo entre ellos, sólo aparece como ventaja de la laparoscopia la menos incidencia de infección de herida operatoria.

Existen subgrupos de pacientes que se verían beneficiados con la técnica laparoscópica como es el paciente obeso, ya que permite explorar toda la cavidad peritoneal por la misma aproximación, sin la necesidad de ampliación de la laparotomía, como frecuentemente ocurre en estos pacientes con la técnica clásica.

Demás es recalcar la experiencia del equipo quirúrgico en técnicas laparoscópicas, que permitirán efectuar la apendicectomía en cualquier variación anatómica en forma segura y expedita.
El trabajo presentado nos muestra un estudio prospectivo, de poblaciones comparables, entre pacientes con apendicitis aguda, que tenían como característica diferencial el tener peso normal y obesidad (IMC mayor a 30). Destaca este hecho, por la prevalencia de la obesidad en nuestro país.

En la introducción se plantea la problemática en forma muy clara y documentada.

Los resultados muestran menores complicaciones de la pared en la técnica laparoscópica; en el resto de los parámetros estudiados no hubo diferencias significativas, lo que es concordante con otros estudios. La principal observación es que la muestra es muy pequeña para sacar conclusiones definitivas.

Es de destacar los bajos tiempos quirúrgicos, lo que demuestra la experiencia de los 2 cirujanos que participaron en el presente estudio.

En conclusión, el trabajo tiene el mérito de ser un estudio prospectivo, y de un tema aún en controversia.

Por los antecedentes anteriormente enunciados, me permito aceptar el trabajo y recomendar la incorporación del Dr. Marcelo Zamorano Díaz, como Miembro Titular de la Sociedad de Cirujanos de Chile.

Dr. Carlos Farías Ortega 